\title{
Regulation of tRNA synthesis and modification in physiological conditions and disease
}

Transfer RNAs (tRNAs) are essential adaptor molecules that convert information contained in mRNA into amino acid sequence. How tRNAs read the genetic code was first proposed by Francis Crick in 1958. tRNA biogenesis, a central pathway essential to all living organisms, has been a subject of interest from the dawn of molecular biology until presentday. tRNA biogenesis involves multiple steps, including synthesis of the initial transcript, maturation of tRNA precursors and charging of tRNA molecules with amino acids. Given the critical role of tRNAs in translation, it is not surprising that cells devote significant resources to tRNA production. Protein complexes involved in tRNA biogenesis have been identified by both genetic and biochemical approaches, which were followed by structural analysis. tRNA maturation and charging occur both in the nucleus and in the cytoplasm, this necessitated that the field explore the cell biology behind tRNA production. This special issue will take us through the tRNA biosynthesis process, with a special emphasis on the regulation of tRNA production by cellular cues.

Process of tRNA synthesis is highly efficient, leading to the production of 3 million tRNAs per generation in the model organism budding yeast, compare that number to the $\sim 60,000$ mRNAs, and one can appreciate the resources committed to produce tRNAs. Because of the energy devoted to tRNA production and because of the required coordination of tRNA and ribosome function, the regulation of tRNA biosynthesis in response to various forms of environmental stress is beneficial for cell economy. For unicellular eukaryotes such as yeast, this process is important for optimal utilization of nutrients and survival. For higher eukaryotes, tRNA production is linked to changes in cell proliferation and cell cycle control. Dysregulation of tRNA biosynthesis leads to a number of pathological states, including cancer. Within the past two decades, there has been much progress in deciphering the multiple layers of control in each step of tRNA biogenesis. In this special issue of BBA-GRM, entitled "Regulation of tRNA synthesis and modifications", 15 review articles from some of the world's leading experts bring together many ideas that drive home the point that the levels of functional tRNAs, which commonly been regarded as abundant "house-keeping" RNAs, are indeed highly regulated.

The special issue opens with a comprehensive overview by Ewan Ramsay and Alessandro Vannini, who cover the dynamic structure of Pol III machinery. This review summarizes the biochemical and structural data on the plasticity of Pol III and its auxiliary factors, TFIIIB and THIIIC, which accounts for regulation each step of tRNA transcription.
The rapid transition from repression to activation of tRNA genes is maintained by the epigenetic state of the genes, controlled by chromatin modifiers. Ashutosh Shukla and Purnima Bhargava describe the role of chromatin structure and nucleosome dynamics in the budding yeast in tRNA transcription. Because they were once thought to be "nucleosome free", the chromatin context of tRNAs genes has received relatively little attention until now.

The control of tRNA synthesis by signaling pathways is achieved by direct targeting of the Pol III complex through phosphorylation and sumoylation of Pol III subunits. The review by Pierre Chymkowitz and Jorrit M. Enserink summarize the relevance of post-translational modifications of Pol III subunits for regulation of tRNA synthesis. Key targets of signaling pathways include the initiation factors, TFIIIB and TFIIIC, and the transcriptional repressor Maf1. TFIIIB and TFIIIC are multi-protein complexes regulated at the level of expression of their individual subunits, as well as through phosphorylation and interaction with partner proteins. Particular aspects of this control in yeast and human cells are described in a review by Damian Graczyk and colleagues.

The transcriptional repressor Maf1 inhibits tRNA synthesis under conditions that are detrimental to cell growth. Ian Willis covers the relationship between Pol III activity and cell physiology, describing various Maf1 phenotypes in different model organisms and cell based systems. Furthermore, Steven Zheng provides insights into the role of Maf1 in growth, aging and oncogenesis, emphasizing the regulation of Pol III by TOR signaling. Importantly, major components of the Maf1 regulatory network are conserved, as illustrated by Celso Benedetti's review on the role of Maf1 in repressing Pol III and suppressing cell growth and proliferation in plants through the TOR pathway.

The final article in this section by Aneeshkumar Arimbasseri analyses interactions between Pol III transcription machinery and tRNA processing factors. In contrast to Pol II system, there is no direct coupling of Pol III transcription and processing. Instead, tRNA processing factors "moonlight" in Pol III transcription and transcription levels indirectly affects tRNA modification machinery.

The second part of this special issue includes articles that address how environmental stresses and cell status can affect the maturation of tRNA precursors and tRNA modifications.

Early steps in tRNA maturation require the chaperone-like La protein, which helps prevent pre-tRNA misfolding and mischanneling into

\footnotetext{
This article is part of a Special Issue entitled: SI: Regulation of tRNA synthesis and modification in physiological conditions and disease edited by Dr. Boguta Magdalena.
} 
offline pathways. The current knowledge on La function and regulation is summarized in the review by Nathan Blewett and Richard Maraia. This article gives unique perspective on differences between yeast and mammals in the subcellular partitioning of pre-tRNA intermediates and differential interactions with La.

tRNA transcription and numerous processing steps occurring in the nucleus are followed by tRNA export to the cytoplasm. The nuclearcytoplasmic traffic of tRNAs is, however, not unidirectional; because tRNA may be also imported back to the nucleus. This tRNA retrograde nuclear import is conserved from budding yeast to vertebrate cells and is stimulated upon nutrient deprivation. A role for intracellular tRNA dynamics is reviewed by Anita Hopper and colleagues, which includes a comprehensive overview of the multiple tRNA nuclear export and import pathways. The subcellular partitioning of factors critical for tRNA function includes the regulation of aminoacyl-tRNA synthetases. The article by Hubert Becker and colleagues covers the subcellular compartments that aminoacyl-tRNA synthetases can occupy in bacteria and eukaryotes, as well as the mechanisms and the signaling pathways that trigger their relocation. Since aminoacyl-tRNA synthetases participate to a wide range of cellular pathways beyond translation, but equally important for cellular homeostasis, the dis-regulation of the subcellular partitioning is associated with the pathologies and diseases.

tRNA modifications are subject to regulation as well. Anders Byström with colleagues review the multistep biosynthesis of wobble base uridines in eukaryotic tRNAs which requires the conserved sixsubunit Elongator complex. Although Elongator has been implicated in several different cellular processes, accumulating evidence suggests that its which in turn, controls translational decoding. A complicated interconnectivity between different enzymatic modification cascades, and their resulting tRNA modifications, are reviewed by Sebastian Glatt and colleagues. As the lack of specific modifications in the anticodon stem and loop causes disrupted proteome homeostasis, diminished response to stress conditions, and the onset of human diseases, the modification cascades have recently gained basic science and clinical interest. Since expression of several microbial virulence factors is induced by changes in environmental conditions, proper control of tRNA modifications have the multifaceted implications for infection and pathogenicity and is important determinant for maintaining cellular function and viability during stress. Regulation of the tRNA modification levels in response to physiological stresses is discussed by Cha San Koh and Peter Sarin. Future development of novel methods and combinatory utilization of existing technologies will bring tRNA modification-mediated regulation of cellular immunity and pathogenicity to the limelight.

Numerous diseases are linked to defective tRNA molecules, which are normally continuously scrutinized and eliminated from cells. Mario Morl and colleagues review recent discoveries that the enzyme responsible for the de novo synthesis of the CCA triplet into the 3'end of tRNAs plays an indispensable role in tRNA quality control by incorporating a second CCA triplet into hypomodified, unstable tRNAs that is recognized as a degradation tag. This final review, gives an update on the latest findings regarding tRNA quality control that turns out to represent an interplay of the CCA-adding enzyme and RNases involved in tRNA degradation and maturation.

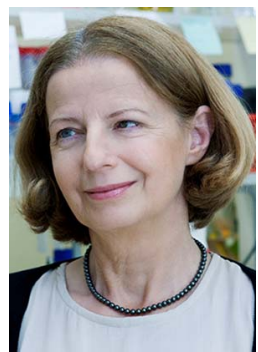

Fot. Magdalena Wiśniewska-Krasińska

Magdalena Boguta received her $\mathrm{PhD}$ in genetics in the Institute of Biochemistry and Biophysics, Polish Academy of Sciences, Poland in 1987. At that time she developed her interest in nuclear-mitochondrial interactions and yeast prions. She spent two years as a postdoctoral fellow in the laboratory of Anita Hopper at Pennsylvania State University, where she started working on tRNA biogenesis in yeast. In 1990 she moved back to Poland and established her own laboratory focused on the control of tRNA gene transcription. In 1999 she went for a sabbatical to the laboratory of Nancy Martin at the University of Louisville. Magdalena Boguta's main discovery is the Maf1 protein, a negative regulator of RNA polymerase III conserved in eukaryotes from yeast to man. Her research focuses on the mode of Maf1 action and the mechanism of the activation of Maf1. She has also investigated the indirect effect of Maf1 on tRNA maturation and decay. She collaborated with Andre Sentenac and Olivier Lefebvre, the leaders in the biochemistry of RNA polymerase III. In 2015 Magdalena Boguta was elected as an EMBO member.

Magdalena Boguta $^{\mathrm{a}, *}$, Joseph C. Reese ${ }^{\mathrm{b}}$

${ }^{a}$ Institute of Biochemistry and Biophysics, Polish Academy of Sciences,

Pawinskiego 5A, 02-106 Warsaw, Poland

${ }^{\mathrm{b}}$ Center for Eukaryotic Gene Regulation, The Pennsylvania State University, United States

E-mail address: magda@ibb.waw.pl

* Corresponding author. 